This item was submitted to Loughborough's Research Repository by the author.

Items in Figshare are protected by copyright, with all rights reserved, unless otherwise indicated.

\title{
Characterization of crude oil and its saturate, aromatic and resin fractions by high-field asymmetric waveform ion mobility spectrometry - high resolution mass spectrometry
}

\section{PLEASE CITE THE PUBLISHED VERSION}

https://doi.org/10.1021/acs.energyfuels.8b02718

\section{PUBLISHER}

(c) American Chemical Society

\section{VERSION}

AM (Accepted Manuscript)

\section{PUBLISHER STATEMENT}

This paper was accepted for publication in the journal Energy and Fuels and the definitive published version is available at https://doi.org/10.1021/acs.energyfuels.8b02718

\section{LICENCE}

CC BY-NC-ND 4.0

\section{REPOSITORY RECORD}

Szykula, Katarzyna, Christianne Wicking, Samuel Whitmarsh, Colin Creaser, and Jim Reynolds. 2019. "Characterization of Crude Oil and Its Saturate, Aromatic and Resin Fractions by High-field Asymmetric Waveform Ion Mobility Spectrometry - High Resolution Mass Spectrometry". figshare.

https://hdl.handle.net/2134/35442. 


\title{
Characterization of crude oil and its saturate,
} aromatic and resin fractions by high-field asymmetric waveform ion mobility spectrometry high resolution mass spectrometry

Katarzyna M. Szykuła, ${ }^{\dagger}$ Christianne Wicking, ${ }^{\ddagger}$ Samuel Whitmarsh ${ }^{\ddagger}$, Colin S. Creaser, ${ }^{\dagger}$ and James C. Reynolds ${ }^{*}+$

†Centre for Analytical Science, Department of Chemistry, Loughborough University, Leicestershire, LE11 3TU, United Kingdom

‡BP Formulated Products Technology, Whitchurch Hill, Pangbourne, Reading, RG8 7QR, United Kingdom

\begin{abstract}
:
High-field asymmetric waveform ion mobility spectrometry (FAIMS) was coupled to a high resolution Orbitrap mass spectrometer (MS) with a heated electrospray ionisation (HESI) source for the analysis of crude oil and respective saturate, aromatic and resin fractions. Four classes of compounds N1, N1S1, O1S1 and O2S1 were investigated using FAIMS 1D compensation field scans from -3 to $5 \mathrm{Td}$ for the crude oil and FAIMS static scans from 0.5 to $2.5 \mathrm{Td}$ with $0.5 \mathrm{Td}$ increments for fractions. In all cases, the incorporation of FAIMS into the analysis resulted in an increased number of detected peaks for both the crude oil and fractions. The most significant change was noticed in the aromatic fraction with an increase of $218 \%$ for $\mathrm{N} 1$ and up to $514 \%$ for O2S1 class of compounds observed. In addition, preanalytical fractionation combined with FAIMS-MS enabled a higher number of molecular features to be observed compared to whole oil for three classes of compounds N1, O1S1 and O2S1 by $19 \%$, 45\% and $83 \%$, respectively.
\end{abstract}




\section{Graphical Abstract:}

FAIMS Off

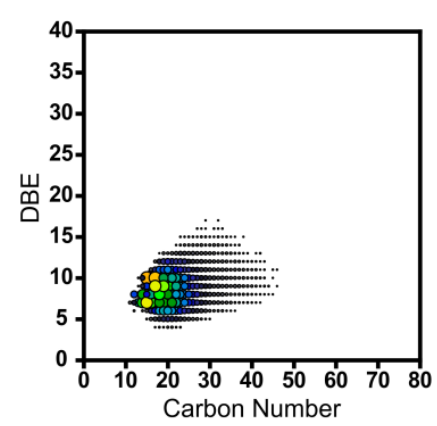

FAIMS On

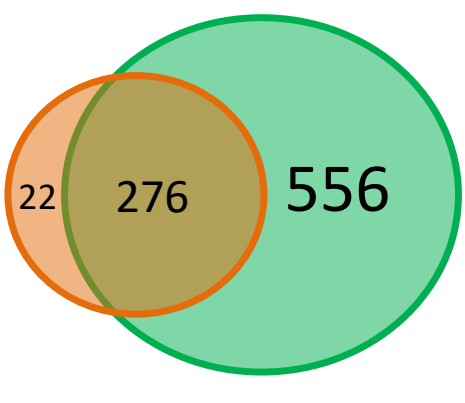

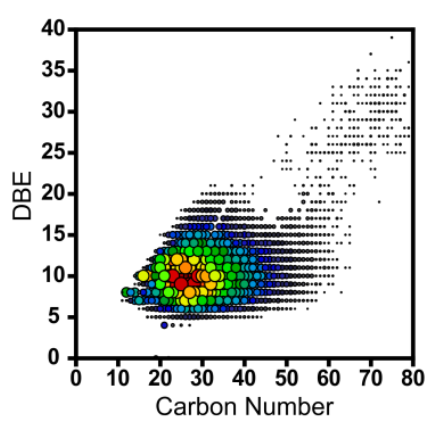

Keywords: crude oil, SARA fractions, field asymmetric waveform ion mobility, high resolution mass spectrometry

\section{Introduction}

Crude oils are considered one of the most analytically complex samples, containing thousands of different compounds, within a dynamic abundance range of $10^{4}-10^{5}$. The complete list of all chemical species in a crude oil is called the petroelome. ${ }^{1-5}$ In order to perform an effective analytical characterization of such a complicated sample, advanced instrumentation is required. High resolution mass spectrometry (HRMS), including ultra-high resolution Fourier Transform mass spectrometers (FTMS) and the Orbitrap are therefore routinely used in the analysis of oils and lubricants. ${ }^{6-9}$ However, even with this advanced technology, the full characterization of crude oils is still a challenge and pre-analysis separation is often required. The most common method being a chromatographic separation into four fractions: saturates, aromatics, resins and asphaltenes, called SARA fractionation, which has been shown to result in the detection of greater numbers of oil compounds compared to unfractionated crude oil. ${ }^{10-13}$

An alternative technique to HRMS that can facilitate the separation of ionized molecules is ion mobility spectrometry (IMS), which utilizes the differences in the velocity of ions while they travel through a drift tube containing a buffer gas when subjected to an electric field. ${ }^{14}$ In petroleomics studies, ion mobility (IM) is mostly used as a complementary technique to 
FTMS, providing additional information to mass-to-charge $(\mathrm{m} / \mathrm{z})$, such as ion mobility and drift time can be used to determine collisional cross section. ${ }^{15-19}$

The first application of IM-MS for crude oil characterization was reported by FernandezLima et al. ${ }^{15}$ Their analysis of crude oils (light, medium and heavy) using laser desorption ionization (LDI) with IM-MS and with FTMS resulted in obtaining fingerprints with size/shape and molecular composition for petroleum crude oils. In 2013, Ponthus et al. ${ }^{16}$ compared the application of travelling wave ion mobility spectrometry-quadrupole-time of flight mass spectrometry (TWIMS-MS) to FTMS for the analysis of a resin fraction from Nigerian Egina oil. Eberlin and co-workers applied TWIMS-MS and ion mobility-quadrupole time-of-flight mass spectrometry (IM-QTOF-MS) for the analysis of crude oils and gasoline with and without additives. ${ }^{17,18}$ With these methods, they obtained a clear separation of contaminants from crude oil constituents as well as gasoline constituents from its additives, which could not be detected via MS alone. Recently, Lalli et al. demonstrated the use of IMTOF-MS for the separation of functional isomers in petroleum emulsion interfacial material. ${ }^{19}$ By applying post-IM collision induced dissociation (CID) they were able to characterize three isomers with $\mathrm{m} / \mathrm{z}$ 325.1830. This was confirmed by FT-ICR-MS where no significant isobars of that $\mathrm{m} / \mathrm{z}$ were observed.

An alternative to drift tube ion mobility spectrometry is high field asymmetric waveform ion mobility spectrometry (FAIMS), also known as differential mobility spectrometry (DMS). This method allows the separation of ions based on their difference in mobility at high and low electric field, instead of using only a low electric field as in conventional IMS. ${ }^{20-22}$ Briefly, ions are carried by a gas flow through a channel between two parallel electrodes. An alternating voltage, which consists of a high-amplitude component for a short period of time followed by a low-amplitude component with a longer time duration, creates the alternating electric field (dispersion field, DF) between the two plates. When an ion's mobility in the high and low field components are the same, ions have a net variance drift of zero in the direction of the DF and they pass through the channel and reach the detector. In the case where the differential mobility under high and low field conditions has a positive or negative net variance, the ions will collide with one of the electrodes, where they are neutralized. In order to counteract this drift towards the electrodes, an additional field (compensation field, $\mathrm{CF}$ ) is applied. The CF compensates only those ions with a specific net drift velocity allowing them to be transmitted. If a sweep of the range of values for the CF is performed, a differential mobility CF spectrum is acquired. 
The applications of FAIMS-MS to crude oil and lubricant analysis has received little attention compared to IM-MS, but has shown its advantage for the reduction of spectral complexity as well as the separation of the isomeric species in a range of different compounds. ${ }^{23-27}$ The first application of FAIMS-MS for the analysis of naphthenic acids (NA) was reported by Gabryelski et al. ${ }^{23}$ Here, standards and naturally occurring naphthenic acid mixtures were analyzed by combining FAIMS with quadrupole, time-of-flight and tandem mass spectrometry. It was shown that ESI-FAIMS-MS is suitable for the characterization of NA in relatively short time (3 minutes) without the need for laborious sample preparation process. Moreover, the combination of FAIMS with tandem MS resulted in more detailed information about NA mixtures than any other technique used in this field of application. The application of differential mobility spectrometry and mass spectrometry (DMS-MS) for the NA analysis was presented by Noestheden et al. ${ }^{25}$ In this work, naphthenic acid fraction components (NAFCs) were separated from oil sands processaffected water (OSPW) within 2 minutes with a minimal signal loss and the method retained the same dynamic range. Furthermore, this method allowed for the separation of potential isomeric species from OSPW extract in seconds, which showed a significant advantage over conventional chromatographic methods. The use of FAIMS-MS for the analysis of the whole crude oils was presented by Schrader et al. ${ }^{24}$ and Vetere $e l$ al. ${ }^{26}$ The first paper describes the coupling of a cylindrical FAIMS device to an LTQ-Orbitrap mass spectrometer fitted with an ESI source for the analysis of nitrogen-rich crude oil and showed the distribution of $\mathrm{m} / \mathrm{z}$ over the acquired CF ranged as well as double bond equivalent (DBE) vs carbon number. This method was able to resolve $\mathrm{NO}$ and $\mathrm{NO}_{2}$ class of compounds, however, the peak resolution of $\mathrm{N}_{1}$ class of compounds was not sufficient, suggesting that the need for a pre-treatment method before the analysis is required. The later work shows the application of photoionization techniques: 1-photon VUV ionization (APPI) and 2-photon ionization with laser radiation (APLI) combined to FAIMS-FTMS for the analysis of heavy North American crude oil. The authors compared the DBE distribution over a CF range for the $\mathrm{S}_{1}$ class of compounds as well as for hydrocarbon class, showing the differences in both methods. Recently, Da Costa et al. ${ }^{27,28}$ have reported the application of FAIMS-MS for the analysis of oils and additives using ESI and desorption electrospray (DESI) ionization. The incorporation of FAIMS improved the additives responses from an oil matrix at low concentrations, which could not be confidently discriminated by MS alone. 
This work combines for the first time a fast scanning miniaturised FAIMS with HRMS, using an Orbitrap mass spectrometer, for the characterization of crude oil and its SAR fractions. We present the results of constituents detected from crude oil before and after fractionation, enabling a direct comparison of the number of N1, N1S1, O1S1 and O2S1 species detected with and without FAIMS.

\section{Experimental section}

2.1. Samples and Reagents. Methanol LC/MS grade, toluene $99+\%$ extra pure, $n$-hexane HPLC grade and chloroform 99+\% extra pure were purchased from Fisher Scientific (Loughborough, UK). Formic acid was purchased from Sigma Aldrich (Gillingham, UK). Basrah Light crude oil was purchased from Onta Inc. (Toronto, Canada). Silica gel was purchased from BDH (Poole, UK).

2.2. Sample preparation. A SARA-type fractionation was performed on a dried silica column (1.2 g of silica, 60-120 mesh, particle size $0.13-0.25 \mathrm{~mm}){ }^{29}$ The column was conditioned with the solvents used for eluting fractions, in reverse order, starting from the most polar mixture. The last part of the n-hexane fraction to elute from the column conditioning was collected as a blank. Basrah Light crude oil (8 to $17 \mathrm{mg}$ ) was dissolved in $50-100 \mu \mathrm{L}$ of $\mathrm{n}$-hexane in order to precipitate asphaltenes before loading the crude oil supernatant onto the column. First, the column was eluted with n-hexane (3.6 mL; saturates fraction). This was followed by the elution with a mixture of n-hexane:toluene (7:3 v/v, 2.4 $\mathrm{mL}$; aromatics fraction). The final fraction was removed from the column by a chloroform:methanol mixture (3:2 v/v, $2.4 \mathrm{~mL}$; resins fraction). After the elution of the resins fraction, the small amount of asphaltenes still retained on the column were eluted with methanol $(1.2 \mathrm{~mL})$. The whole fractionation process was repeated 4 times and the fractions were pooled, as well as the blank samples, and evaporated to dryness. The crude oil and its fractions were dissolved in a mixture of methanol:toluene $(6: 4+0.1 \% \mathrm{v} / \mathrm{v}$ formic acid) to a final concentration of $0.5 \mathrm{mg} \mathrm{mL}^{-1}$. Solutions were infused directly into the HESI source of the mass spectrometer at flow rate of $100 \mu \mathrm{L} \mathrm{m^{-1 }}$.

2.3. Instrumentation. The crude oil and fractions were analysed by HESI-FAIMS-MS using a HESI-II Probe and a Thermo Orbitrap Q Exactive Plus mass spectrometer (Thermo Fisher Scientific, Massachusetts, USA) coupled with a miniaturized Owlstone ultraFAIMS chip (Owlstone Ltd., Cambridge, UK) operated at ambient pressure. The analysis was performed in positive ion mode with the following parameters: sheath gas (nitrogen) flow rate 10 
(arbitrary units), HESI temperature $250{ }^{\circ} \mathrm{C}$, capillary temperature $300{ }^{\circ} \mathrm{C}$, spray voltage 4.0 $\mathrm{kV}$, AGC target 1e6, mass range $m / z 150-1100$ and resolution 280,000 at $\mathrm{m} / \mathrm{z}$ 200. FAIMS chip parameters were as follows: $100 \mu \mathrm{m}$ electrode gap, trench length (span) $78.1 \mathrm{~mm}$ and ion path length $700 \mu \mathrm{m}$. The chip temperature was set at $150{ }^{\circ} \mathrm{C}$. Data acquisition was performed using a fixed DF at $250 \mathrm{Td}$, with $1 \mathrm{D}$ mode scanning in the CF range from -3.0 to 5.0 Td with a sweep duration of 60 seconds for the crude oil, and with static mode at CFs from 0.5 to $2.5 \mathrm{Td}$ in $0.5 \mathrm{Td}$ increments with an acquisition time 30 seconds for fractions and the blank. Data were processed using PetroORG software (Florida State University) to obtain peak lists and plots of DBE vs carbon number. Heatmaps and mass spectra were generated by Matlab R2016a (MathWorks, Inc, Natick, Massachusetts) and 3D plot by OriginLab 2015 (Academic version, b9.2.272).

\section{Results and Discussion}

3.1. Crude oil. Basrah Light crude oil was analysed by HESI-MS and HESI-FAIMS-MS in order to compare the two techniques. A 1D FAIMS scan was performed in the CF range from -3.0 to $5.0 \mathrm{Td}$, at a fixed DF of $250 \mathrm{Td}$ (Figure 1). The FAIMS-MS analysis shows ions transmitted in the CF range $0.5-2.5 \mathrm{Td}$, associated with the ionized molecules from the crude oil. The peak is baseline resolved from a lower intensity feature centred at $0 \mathrm{Td}$ assigned to solvent-based clusters and multimers. This results in two distinctive peaks displayed on the heatmap (Figure 1a) and 3D plot (Figure 1b). The $m / z$ distribution for the FAIMS scans shows that the highest intensity was recorded for the $m / z$ range 250 - 400 . 
a)

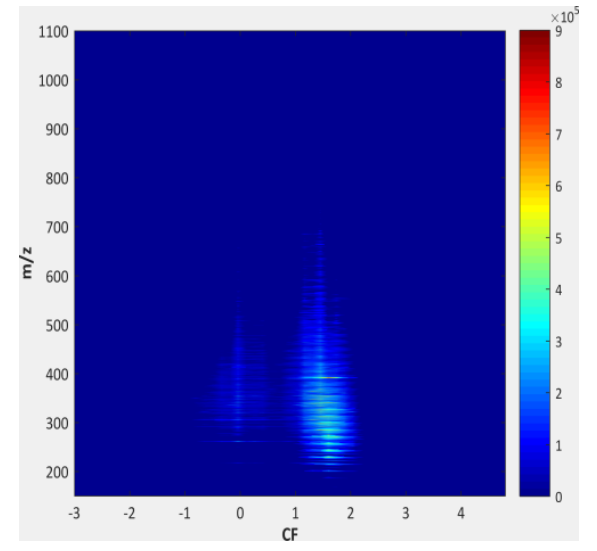

b)

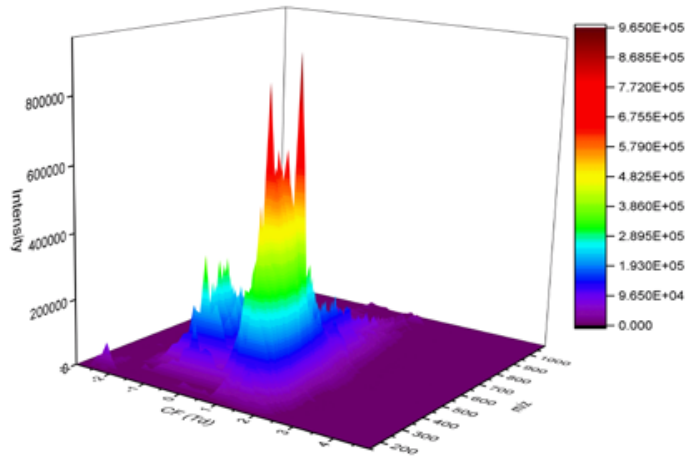

Figure 1. HESI-FAIMS-MS analysis of Basrah Light crude oil, a) heatmap presenting $\mathrm{m} / \mathrm{z}$ vs CF, c) 3D plot.

The number of features assigned by PetroORG for four classes of compounds $\mathrm{N} 1\left(\mathrm{C}_{\mathrm{x}} \mathrm{H}_{\mathrm{y}} \mathrm{N}\right)$, N1S1 $\left(\mathrm{C}_{\mathrm{x}} \mathrm{H}_{\mathrm{y}} \mathrm{NS}\right)$, O1S1 $\left(\mathrm{C}_{\mathrm{x}} \mathrm{H}_{\mathrm{y}} \mathrm{SO}\right)$ and O2S1 $\left(\mathrm{C}_{\mathrm{x}} \mathrm{H}_{\mathrm{y}} \mathrm{SO}_{2}\right)$ for crude oil acquired by HESI-MS and HESI-FAIMS-MS are presented in Table 1. For the most abundant N1 class, 946 peaks were detected by HESI-MS and 1214 by the incorporation of FAIMS into the analysis. This shows a 22\% increase in identified peaks. Moreover, as 912 features were common to both analytical methods, there were 302 unique peaks detected only by FAIMS-MS (Figure 2). This indicates how much additional information about the sample can be obtained by applying FAIMS separation, which could not be obtained by using mass spectrometry alone.

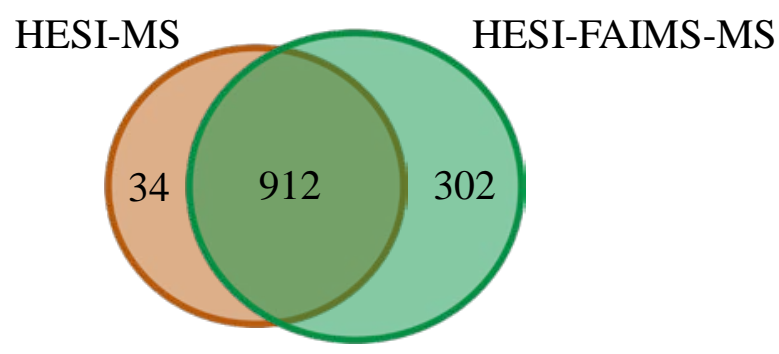

Figure 2. Venn diagram with the number of peaks assigned by PetroORG for the N1 class of compounds for Basrah Light crude oil. On the left HESI-MS (orange), on the right HESI-FAIMS-MS (green).

A similar trend was observed for the N1S1, O1S1 and O2S1 species, with the most significant increase noted for the N1S1 and O1S1 classes of compounds. The peaks identified for those classes increased by $74 \%$ and $84 \%$, respectively.

3.2. Fractions. The crude oil was separated into fractions on a silica column and eluted with different solvents as described earlier. Asphaltenes were precipitated with n-hexane before on-silica fractionation and were not further analysed. Saturate, aromatic and resin fractions 
were analysed by HESI-MS and HESI-FAIMS-MS in static mode, at fixed CFs in the range from 0.5 to $2.5 \mathrm{Td}$, in $0.5 \mathrm{Td}$ increments, at DF of $250 \mathrm{Td}$.

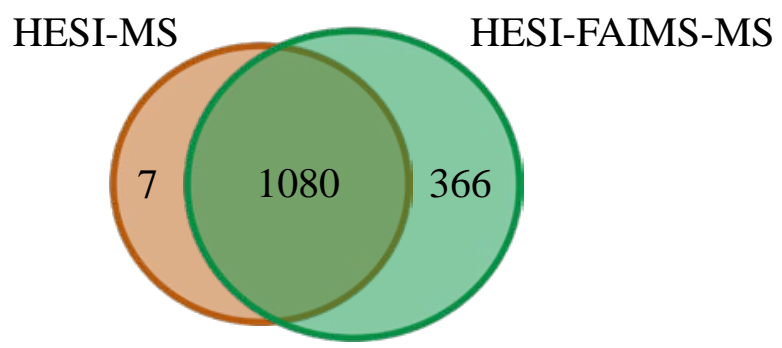

Figure 3. Venn diagram with the number of peaks assigned by PetroORG for the N1 class of compounds for combined fractions. On the left HESI-MS (orange), on the right HESI-FAIMS-MS (green).

The number of molecular features assigned by PetroORG for N1, N1S1, O1S1 and O2S1 classes from saturate, aromatic and resin fractions is compared with and without the application of FAIMS in Table 1. Additionally, the features from all fractions were combined and duplicates were removed for the comparison with the number of features obtained from the crude oil. In this case, as with the unfractionated oil, the N1 class is the most abundant resulting in 1087 peaks without ion mobility separation and 1446 with FAIMS separation. Furthermore, there were 366 peaks detected uniquely by HESI-FAIMS-MS compared to only 7 unique peaks detected by HESI-MS (Figure 3) but not by HESI-FAIMS-MS. The 7 peaks that were not observed by FAIMS-MS (Fig. 3), and the 34 in the analysis of the unfractionated crude oil, were low abundance features, the majority with $\mathrm{m} / \mathrm{z}$ above 500 . Their absence in the FAIMS-MS analysis could be due to lower sensitivity resulting from ion losses when the FAIMS is switched on, resulting in ion intensities falling below the limit of detection. 
Table 1. Molecular features assigned by PetroORG from Basrah Light crude oil and its fractions analysed by HESI-MS (FAIMS-Off) and HESI-FAIMS-MS (FAIMS-On).

\begin{tabular}{|c|c|c|c|c|c|}
\hline \multicolumn{6}{|c|}{ N1 } \\
\hline & Saturates & Aromatics & Resins & $\begin{array}{l}\text { All } \\
\text { fractions* }\end{array}$ & Crude oil ${ }^{* *}$ \\
\hline FAIMS Off & 595 & 298 & 1002 & 1087 & 946 \\
\hline FAIMS On & 1056 & 948 & 1224 & 1446 & 1214 \\
\hline \multicolumn{6}{|c|}{ N1S1 } \\
\hline & Saturates & Aromatics & Resins & $\begin{array}{l}\text { All } \\
\text { fractions* }\end{array}$ & Crude oil** \\
\hline FAIMS Off & 305 & 126 & 328 & 378 & 432 \\
\hline FAIMS On & 598 & 495 & 437 & 702 & 752 \\
\hline \multicolumn{6}{|c|}{ O1S1 } \\
\hline & Saturates & Aromatics & Resins & $\begin{array}{l}\text { All } \\
\text { fractions* }\end{array}$ & Crude oil ${ }^{* *}$ \\
\hline FAIMS Off & 364 & 191 & 236 & 449 & 350 \\
\hline FAIMS On & 818 & 654 & 405 & 935 & 645 \\
\hline \multicolumn{6}{|c|}{ O2S1 } \\
\hline & Saturates & Aromatics & Resins & $\begin{array}{l}\text { All } \\
\text { fractions* }\end{array}$ & Crude oil ${ }^{* *}$ \\
\hline FAIMS Off & 408 & 111 & 425 & 570 & 456 \\
\hline FAIMS On & 686 & 682 & 577 & 878 & 481 \\
\hline
\end{tabular}

*Molecular features combined from all three fractions without duplicates (CF $0.5-2.5 \mathrm{Td}$ ).

**The whole crude oil scanned at CF range from 0.5 to $2.5 \mathrm{Td}$. 

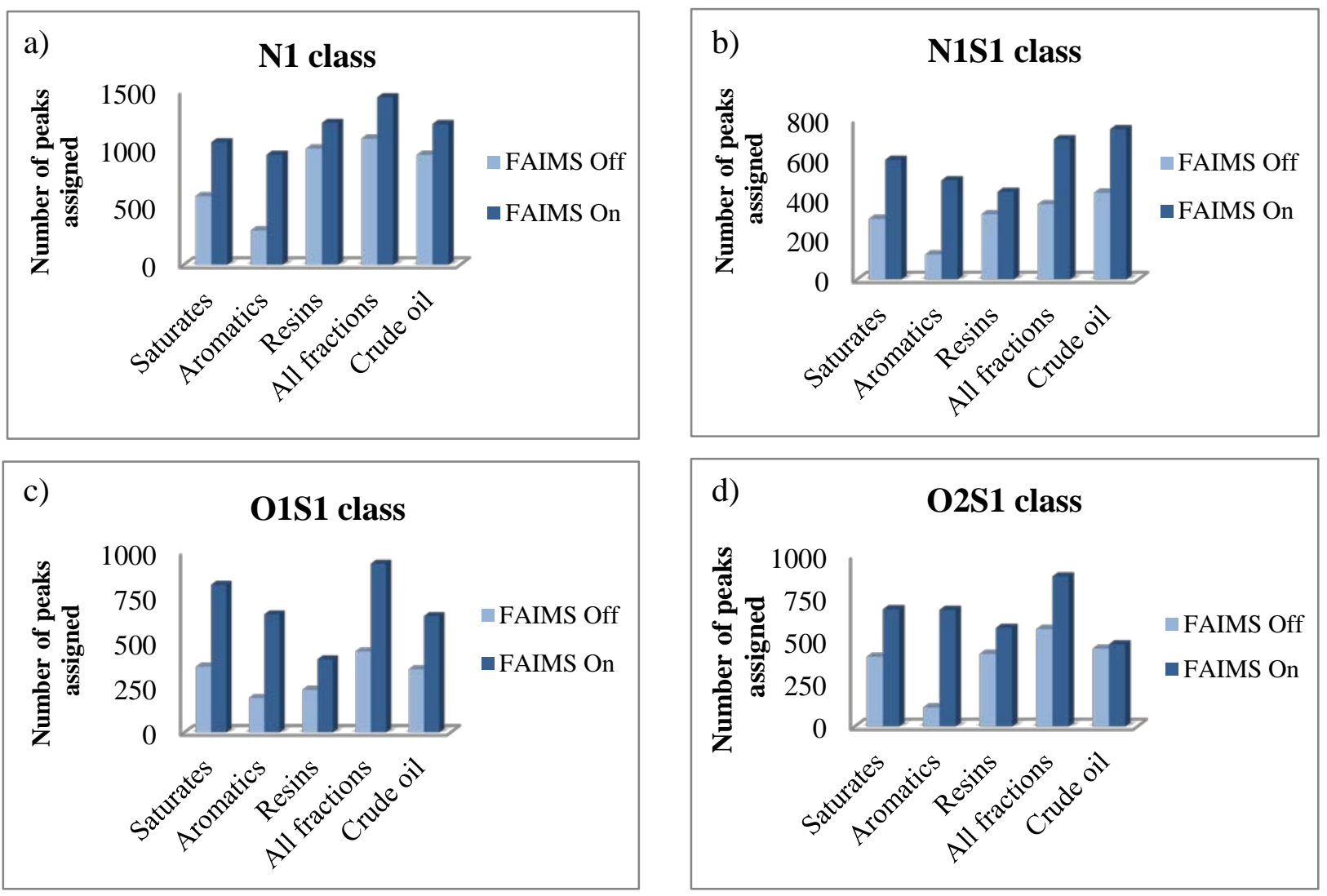

Figure 4. Number of peaks assigned for fractions and crude oil with and without FAIMS; a) N1 class of compounds, b) N1S1 class of compounds, c) O1S1 class of compounds, d) O2S1 class of compounds.

The hyphenation of FAIMS to MS analysis resulted in an increased number of peaks detected for all three fractions, demonstrating the benefit of combining fractionation and FAIMS with HRMS for the characterization of crude oils. Interestingly, the greatest effect on applying FAIMS was observed in the aromatic fraction for every class of compounds under investigation. Here, the number of formulas assigned increased by $218 \%$ for the N1 class with the highest percentage increase of 514\% observed for the O2S1 class of compounds. The incorporation of FAIMS into the analysis results in a reduced noise level and by that the signal-to-noise ratio for low abundance species was raised above the threshold that enables PetroORG to detect these compounds. This effect is especially noticeable in the low abundance species such as the O2S1 class. Moreover, features in the high DBE range respond particularly well to FAIMS, the aromatic fraction contains many high DBE/carbon number species and as a result of the high level of structural diversity in the Basrah Light oil (Figure S1), a considerable increase in the number of peaks detected was observed. 
The application of FAIMS not only increases the number of peaks detected, but also affects the sample profile in general (Figure 5 and Figure 6). Figure 5 presents the plots of DBE vs carbon number for $\mathrm{N} 1$ class for the aromatic fraction with and without FAIMS analysis. It can be clearly seen, that many more peaks were detected with a FAIMS separation compared to HRMS alone. Moreover, the centre of the sample profile has moved to higher DBE and carbon number as well, with a wider intensity profile observed. Without FAIMS applied, the highest intensity was observed for peaks with lower carbon number than 20. However, this moved towards carbon number 25 at CF 0.5 Td. Low intensity peaks with a higher carbon number (over 60) and DBE (>20) are also observed with FAIMS-on.

a)

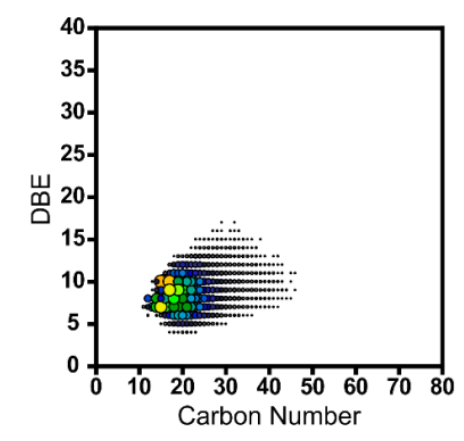

b)

\section{FAIMS On}

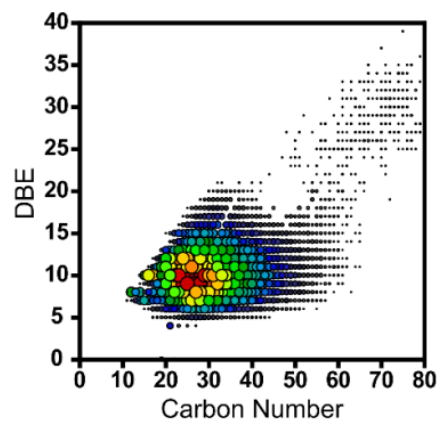

Figure 5. Plots of DBE vs carbon number for N1 class from aromatic fraction, a) without FAIMS, b) with FAIMS, at CF $0.5 \mathrm{Td}$.

The effect of applying different compensation fields on the profile is shown on Figure 6 . Here, plots of DBE vs carbon number as well as mass spectra recorded with and without FAIMS for the N1 class from the saturates fraction are presented. The HESI-MS analysis resulted in obtaining 595 assigned formulas with the highest intensity recorded between $\mathrm{m} / \mathrm{z}$ 200 and 350 and carbon number range of 15-30 (Figure 6a). Noticeable changes can be observed in the DBE vs C plot with HESI-FAIMS-MS analysis at CF $1.0 \mathrm{Td}$, such as the presence of ions with carbon number higher than 60. Moreover, the abundance of the peaks with carbon number between 30 and 45 has increased (Figure 6b). MS profile broadened at CF 1.0 Td showing the higher intensity for the ions with $\mathrm{m} / \mathrm{z}$ greater than 400 . Increasing the CF to $2.5 \mathrm{Td}$ resulted in two separate distributions being observed (Figure 6c). The first, smaller distribution, with its centre at $m / z 250$ and the larger one with the midpoint located at $\mathrm{m} / \mathrm{z}$ 500. These two profiles can also be distinguished on the Kendrick plot at carbon number 20 and 35. These distributions are masked in the first plot and it is only through the use of FAIMS they can be efficiently visualized. 
The implementation of the pre-fractionation step before the analysis also resulted in increasing the number of detected peaks for the following class of compounds: N1, O1S1 and O2S1 (Table 1). It is especially noticeable for S1O2 class of compounds, where 391 more peaks were found in fractions than in the crude oil. The only class that shows the opposite effect is N1S1, where 54 and 52 more peaks where detected in crude oil than in fractions, without and with FAIMS, respectively, possibly due to high mass NS class compounds being unresolved from the $\mathrm{N}$ class at higher masses in the Orbitrap. 
a)

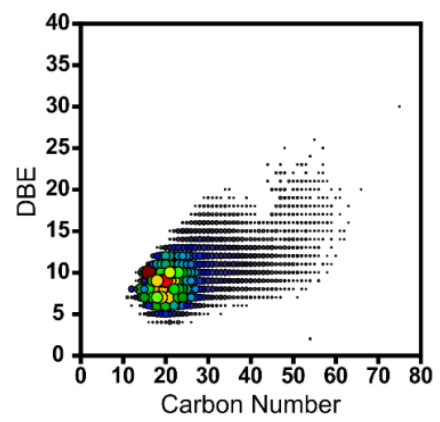

FAIMS Off

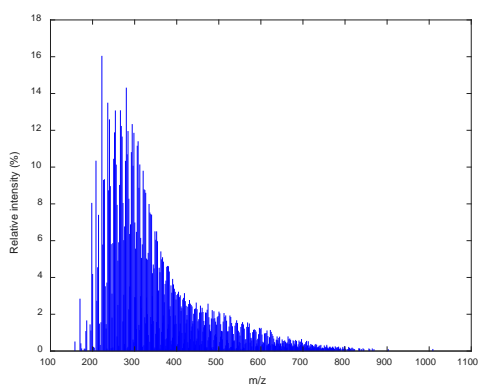

b)

FAIMS On CF $1.0 \mathrm{Td}$
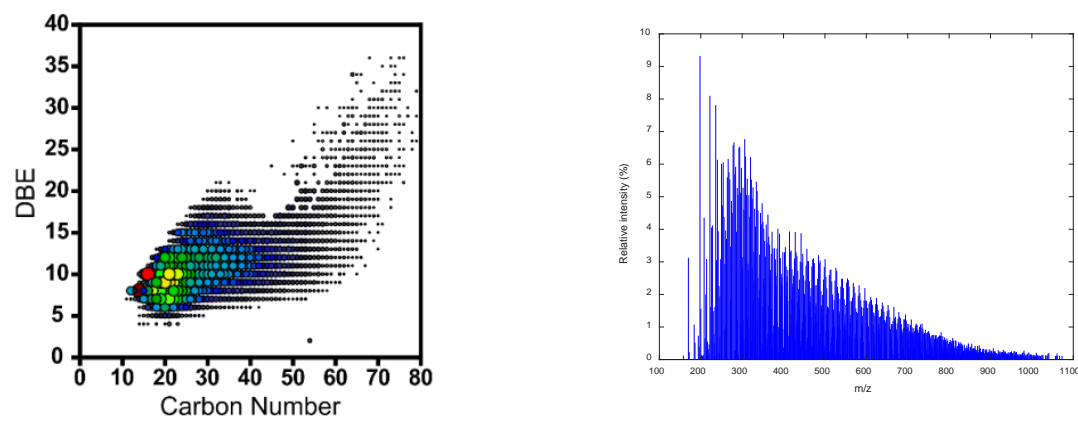

c)

FAIMS On CF 2.5 Td
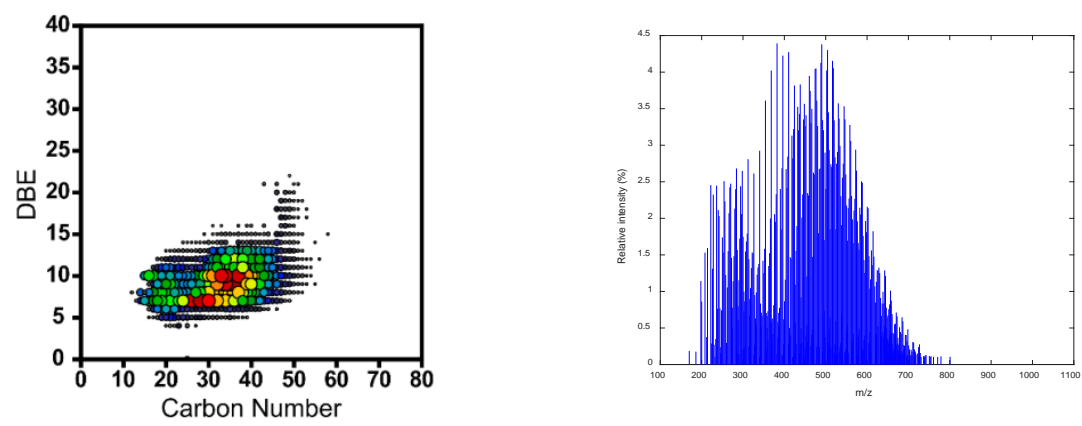

Figure 6. Plots of DBE vs carbon number and mass spectra for N1 class from saturate fraction, a) without FAIMS, b) at CF $1.0 \mathrm{Td}$, c) at CF $2.5 \mathrm{Td}$.

\section{Conclusion}

Miniaturised FAIMS combined with HRMS was applied to the analysis of Basrah Light crude oil and its saturate, aromatic and resin fractions compared to HRMS alone. The implementation of FAIMS as an additional separation based on differential mobility resulted in an increased number of molecular features detected in the crude oil and fractions by reducing the noise level and thus extending the dynamic range. This increase was particularly observable for aromatic hydrocarbons where 514\% more peaks were detected with FAIMS 
for O2S1 class of compounds. Scanning through the range of compensation fields changes the MS and PetroOrg profiles of the sample giving additional information, which could not be obtained by mass separation alone. Pre-analysis separation of the crude oil into fractions provided the higher number of assigned peaks for the N1, O1S1 and O2S1 classes of compounds. The overall increase in the number of constituents observed by combining SAR fractionation with FAIMS pre-selection and HRMS has significant potential in oil characterization and petroleomic studies.

\section{Acknowledgements}

Authors would like to thank Owlstone Ltd. for the financial and technical support and BP for the technical support.

\section{References}

(1) Oliver C. Mullins, Eric Y. Sheu, Ahmed Hammami, A. G. M. Asphaltenes, Heavy Oils, and Petroleomics; Springer, 2007.

(2) Panda, S. K.; Andersson, J. T.; Schrader, W. Characterization of Supercomplex Crude Oil Mixtures: What Is Really in There? Angew. Chemie Int. Ed. 2009, 48 (10), 1788-1791.

(3) Christine A. Hughey; Ryan P. Rodgers, A.; Alan G. Marshall. Resolution of 11000 Compositionally Distinct Components in a Single Electrospray Ionization Fourier Transform Ion Cyclotron Resonance Mass Spectrum of Crude Oil. Anal. Chem. 2002, 74 (16), 41454149.

(4) Alan G. Marshall and; Ryan P. Rodgers†. Petroleomics: The Next Grand Challenge for Chemical Analysis. Acc. Chem. Res. 2004, 37 (1), 53-59.

(5) Marshall, A. G.; Rodgers, R. P. Petroleomics: Chemistry of the Underworld. Proc. Natl. Acad. Sci. U. S. A. 2008, 105 (47), 18090-18095.

(6) Zhurov, K. O.; Kozhinov, A. N.; Tsybin, Y. O. Evaluation of High-Field Orbitrap Fourier Transform Mass Spectrometer for Petroleomics. Energy \& Fuels 2013, 27 (6), 2974-2983.

(7) Griffiths, M. T.; Da Campo, R.; O’Connor, P. B.; Barrow, M. P. Throwing Light on Petroleum: Simulated Exposure of Crude Oil to Sunlight and Characterization Using Atmospheric Pressure Photoionization Fourier Transform Ion Cyclotron Resonance Mass Spectrometry. Anal. Chem. 2014, 86 (1), 527-534. 
(8) Gaspar, A.; Schrader, W. Expanding the Data Depth for the Analysis of Complex Crude Oil Samples by Fourier Transform Ion Cyclotron Resonance Mass Spectrometry Using the Spectral Stitching Method. Rapid Commun. Mass Spectrom. 2012, 26 (9), 1047-1052.

(9) Corilo, Y. E.; Vaz, B. G.; Simas, R. C.; Lopes Nascimento, H. D.; Klitzke, C. F.; Pereira, R. C. L.; Bastos, W. L.; Santos Neto, E. V.; Rodgers, R. P.; Eberlin, M. N. Petroleomics by EASI( \pm ) FT-ICR MS. Anal. Chem. 2010, 82 (10), 3990-3996.

(10) Klein, G. C.; Angström, A.; Ryan P. Rodgers, A.; Alan G. Marshall. Use of Saturates/Aromatics/Resins/Asphaltenes (SARA) Fractionation To Determine Matrix Effects in Crude Oil Analysis by Electrospray Ionization Fourier Transform Ion Cyclotron Resonance Mass Spectrometry. 2006.

(11) Shi, Q.; Hou, D.; Chung, K. H.; Xu, C.; Zhao, S.; Zhang, Y. Characterization of Heteroatom Compounds in a Crude Oil and Its Saturates, Aromatics, Resins, and Asphaltenes (SARA) and Non-Basic Nitrogen Fractions Analyzed by Negative-Ion Electrospray Ionization Fourier Transform Ion Cyclotron Resonance Mass Spectrometry. Energy \& Fuels 2010, 24 (4), 25452553.

(12) Cho, Y.; Na, J.-G.; Nho, N.-S.; Kim, S.; Kim, S. Application of Saturates, Aromatics, Resins, and Asphaltenes Crude Oil Fractionation for Detailed Chemical Characterization of Heavy Crude Oils by Fourier Transform Ion Cyclotron Resonance Mass Spectrometry Equipped with Atmospheric Pressure Photoionization. Energy \& Fuels 2012, 26 (5), 2558-2565.

(13) Gaspar, A.; Zellermann, E.; Lababidi, S.; Reece, J.; Schrader, W. Characterization of Saturates, Aromatics, Resins, and Asphaltenes Heavy Crude Oil Fractions by Atmospheric Pressure Laser Ionization Fourier Transform Ion Cyclotron Resonance Mass Spectrometry. Energy \& Fuels 2012, 26 (6), 3481-3487.

(14) Eiceman, G. A.; Karpas, Z.; Hill, H. H. Ion Mobility Spectrometry, Third.; CRC Press, 2016.

(15) Fernandez-Lima, F. A.; Becker, C.; McKenna, A. M.; Rodgers, R. P.; Marshall, A. G.; Russell, D. H. Petroleum Crude Oil Characterization by IMS-MS and FTICR MS. Anal. Chem. 2009, 81 (24), 9941-9947.

(16) Ponthus, J.; Riches, E. Evaluating the Multiple Benefits Offered by Ion Mobility-Mass Spectrometry in Oil and Petroleum Analysis. Int. J. Ion Mobil. Spectrom. 2013, 16 (2), 95103.

(17) Fasciotti, M.; Lalli, P. M.; Klitzke, C. F.; Corilo, Y. E.; Pudenzi, M. A.; Pereira, R. C. L.; Bastos, W.; Daroda, R. J.; Eberlin, M. N. Petroleomics by Traveling Wave Ion Mobility-Mass 
Spectrometry Using $\mathrm{CO}_{2}$ as a Drift Gas. Energy \& Fuels 2013, 27 (12), 7277-7286.

(18) Santos, J. M.; Galaverna, R. de S.; Pudenzi, M. A.; Schmidt, E. M.; Sanders, N. L.; Kurulugama, R. T.; Mordehai, A.; Stafford, G. C.; Wisniewski, A.; Eberlin, M. N. Petroleomics by Ion Mobility Mass Spectrometry: Resolution and Characterization of Contaminants and Additives in Crude Oils and Petrofuels. Anal. Methods 2015, 7 (11), 44504463.

(19) Lalli, P. M.; Jarvis, J. M.; Marshall, A. G.; Rodgers, R. P. Functional Isomers in Petroleum Emulsion Interfacial Material Revealed by Ion Mobility Mass Spectrometry and CollisionInduced Dissociation. Energy \& Fuels 2017, 31 (1), 311-318.

(20) Buryakov, I. A.; Krylov, E. V.; Nazarov, E. G.; Rasulev, U. K. A New Method of Separation of Multi-Atomic Ions by Mobility at Atmospheric Pressure Using a High-Frequency Amplitude-Asymmetric Strong Electric Field. Int. J. Mass Spectrom. Ion Process. 1993, 128 (3), 143-148.

(21) Shvartsburg, A. A. Differential Ion Mobility Spectrometry: Nonlinear Ion Transport and Fundamentals of FAIMS; CRC Press, 2008.

(22) Pollard, M. J.; Hilton, C. K.; Li, H.; Kaplan, K.; Yost, R. A.; Hill, H. H. Ion Mobility Spectrometer-Field Asymmetric Ion Mobility Spectrometer-Mass Spectrometry. Int. J. Ion Mobil. Spectrom. 2011, 14 (1), 15-22.

(23) Gabryelski, W.; Froese, K. L. Characterization of Naphthenic Acids by Electrospray Ionization High-Field Asymmetric Waveform Ion Mobility Spectrometry Mass Spectrometry. Anal. Chem. 2003, 75 (17), 4612-4623.

(24) Schrader, W.; Xuan, Y.; Gaspar, A. Studying Ultra-Complex Crude Oil Mixtures by Using High-Field Asymmetric Waveform Ion Mobility Spectrometry (FAIMS) Coupled to an Electrospray Ionisation-LTQ-Orbitrap Mass Spectrometer. Eur. J. Mass Spectrom. 2014, 20 (1), 43-49.

(25) Noestheden, M. R.; Headley, J. V.; Peru, K. M.; Barrow, M. P.; Burton, L. L.; Sakuma, T.; Winkler, P.; Campbell, J. L. Rapid Characterization of Naphthenic Acids Using Differential Mobility Spectrometry and Mass Spectrometry. Environ. Sci. Technol. 2014, 48 (17), 1026410272.

(26) Vetere, A.; Schrader, W. 1- and 2-Photon Ionization for Online FAIMS-FTMS Coupling Allows New Insights into the Constitution of Crude Oils. Anal. Chem. 2015, 87 (17), 88748879. 
(27) Da Costa, C.; Turner, M.; Reynolds, J. C.; Whitmarsh, S.; Lynch, T.; Creaser, C. S. Direct Analysis of Oil Additives by High-Field Asymmetric Waveform Ion Mobility SpectrometryMass Spectrometry Combined with Electrospray Ionization and Desorption Electrospray Ionization. Anal. Chem. 2016, 88 (4), 2453-2458.

(28) Da Costa, C. Applications of Desorption Electrospray Ionisation Mass Spectrometry and Ion Mobility Spectrometry to Petroleomic and Lubricant Analysis, Loughborough University, 2015.

(29) Tijjani, I. M. N.; Dioha, I. J.; Musa, A.; Sale, H.; Lawal, A. M. SARA Separation and Determination of Concentration Levels of Some Heavy Metals in Organic Fractions of Nigerian Crude Oil. Chem. Mater. Res. 2013, 3 (4), 7-15. 


\section{Characterization of crude oil and its saturate,}

aromatic and resin fractions by high-field asymmetric waveform ion mobility spectrometry high resolution mass spectrometry

Katarzyna M. Szykuła, ${ }^{\dagger}$ Christianne Wicking, ${ }^{\ddagger}$ Samuel Whitmarsh ${ }^{\ddagger}$, Colin S. Creaser, ${ }^{\dagger}$ and James C. Reynolds* ${ }^{*}+$

†Centre for Analytical Science, Department of Chemistry, Loughborough University, Leicestershire, LE11 3TU, United Kingdom

‡BP Formulated Products Technology, Whitchurch Hill, Pangbourne, Reading, RG8 7QR, United Kingdom 


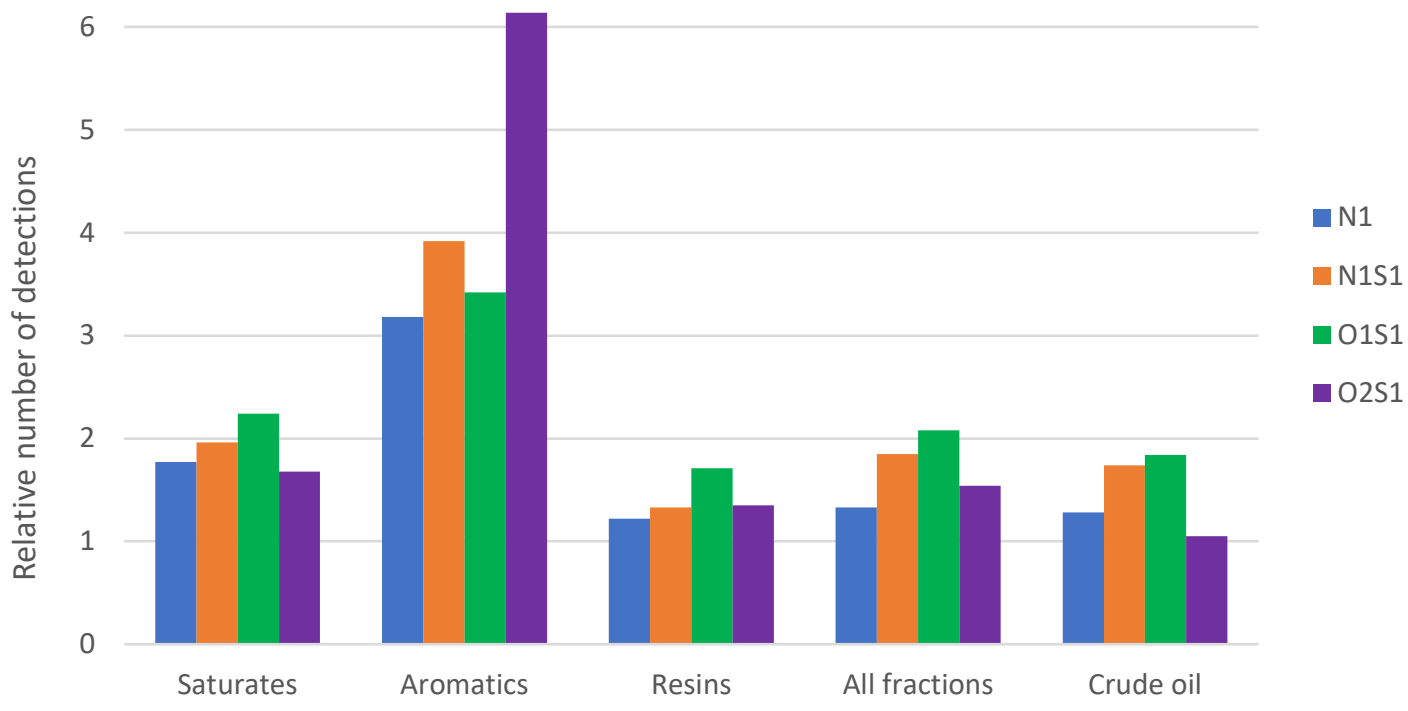

Supporting Figure 1. Relative increase in the number of components detected (FAIMS on/FAIMS off) in the saturates, aromatics and resin fractions, and the unfractionated and crude oil, for the N1, N1S1, O1S1 and O2S1 classes of compounds. 